\title{
Message Transfer in Virtual Reality Simulation of Fire Control
}

\author{
Bin Shen*, Yuan Liu and Guoji Cai \\ Wuhan Donghu University, China, 430212 \\ ${ }^{*}$ Corresponding author
}

\begin{abstract}
A type of equipment fire control system is the core component of the equipment. Real training is often limited by equipment cost, training site, training weather, personnel quality and so on. This requires pre virtual training before the actual operation. In this control system, how to realize the various functions of the system is the core issue of the project. Virtools has the characteristics of strong interaction, good portability and low operating environment requirements. Combining with the practice of training, this paper presents the development of equipment control simulation system based on Virtools, and starts from the design of the Virtools software, and introduces the transmission of the control part of the message.
\end{abstract}

Keywords—simulation; VIRTOOLS; virtual reality; control

\section{INTRODUCTION}

A type of equipment fire control system is the core component of the equipment. Real training is often limited by equipment cost, training site, training weather, personnel quality and so on. This requires pre virtual training before the actual operation. The development of virtual reality simulation platform can help to change this situation.

The virtual reality development software Virtools, issued by Dassault company of France, is a real time 3D environment virtual reality editing software with rich interactive behavior modules. It can produce many different uses of 3D products, such as Internet, computer games, multimedia, education and training, simulation and product display, and so on. It has the advantages of powerful, high picture quality, rich interface and supporting many hardware. Simulation training is an important link to train the practice ability and innovation spirit of the user. As the important equipment of this equipment, the fire control system is very important. Therefore, we have developed the virtual experiment teaching platform of the fire control system according to the teaching content, which is an effective way to enrich the training means.

With the rapid development of computer simulation technology, virtual reality technology has been widely applied in equipment training and support. The Virtools software developed by Dassault France has the characteristics of strong interactivity, good portability and low operating environment. A good virtual reality training system will be established by using Virtools software. In the construction of the software, message passing of control system is the key. Starting from the bottom design of Virtools software, this paper introduces the transmission of control part messages[1-8].

\section{The Overall Design of The System}

The system uses Visual $\mathrm{C}++6.0$ and the Virtools of Dassault company of France as the development platform of the system. Using Solidworks 3D design software and 3dsmax as modeling and animation generating tools, Access for the system database to store sample parts library, special tool library, testing tool library and some industry samples.

Equipment training simulation is an omni directional simulation under virtual environment. It is closely related to the theme of equipment training, and at the same time, throughout the whole life cycle of the product. Equipment training simulation technology includes messaging, conflict resolution and other underlying technologies, equipment operation simulation and environmental simulation technology. Fire control simulation completes a series of action simulation based on the predetermined operation process, including virtual target detection, virtual parameter input, virtual control and so on. The system must complete the interaction between virtual tools and virtual prototypes through the message group. According to the configuration of virtual control simulation environment, it can be divided into immersive virtual control simulation technology and non immersive control simulation technology. This paper mainly studies the non immersive simulation technology by using Virtools 5 software combined with the constructed 3D model[9-13].

The equipment model itself has a large number of parts. Generally, it belongs to large assembly. The 3D modeling technology provided by Solidworks software has a good effect in dealing with large assembly. The system is built on the basis of Solidworks software, and then rendered by 3dsmax software, and then imported into Virtools software for overall simulation design. Virtools software is the key to processing control technology. Its characteristics are that it can be combined with $\mathrm{C}++$, and use VSL to develop flexible control programs. VSL is a very creative scripting language provided by Virtools. It is programmed and developed in the way of transmission and write code, which is different from the existing graphical programming interface of Virtools. It improves the readability and efficiency of the program. It has a good presence, such as the tiger's wings. With this way of development, you will greatly reduce the number of connections and improve the efficiency of program development. This system makes full use of the characteristics of VSL language, reasonably controls message transmission, and designs fire control simulation system flexibly. It meets the requirements of the user. Figure 1 
is the interface of the fire control system. The left side window in the picture is a simulated interface of the sight, and the right side is the simulation interface of the firepower system. There are aiming scales and important signs in the sight lens interface. The content of the logo is one-to-one correspondence to the type of data on the right side of the graph. There is a one-to-one correspondence between the sight field of the sighting telescope and the right data in the graph, all of which require message passing.

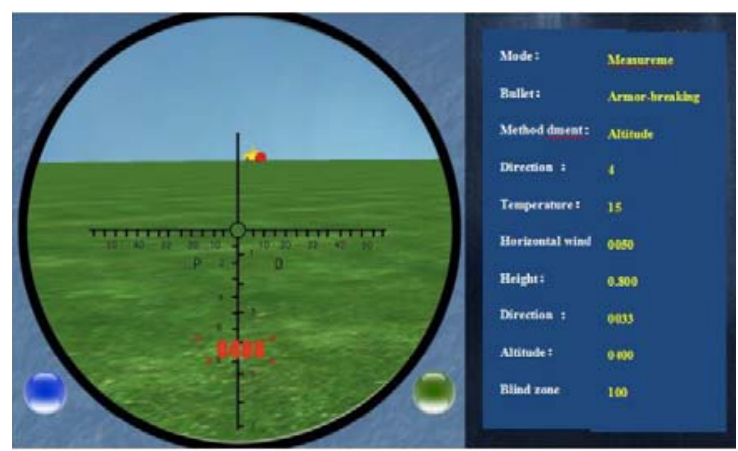

FIGURE I. MAIN INTERFACE OF FIRE CONTROL SYSTEM

\section{MEssage Transmission OF the System}

The system completes a variety of control simulation through a large number of message passing. The bottom of message passing is the construction of system coordinate system. The generation of the operating environment should be based on the assumed equipment configuration point, the 3D scene is built with 3dsmax, and is implanted into the virtual reality, with the necessary lighting, and the camera. The corresponding 3D scene is shown in Figure 1. The behavior interaction module Keyboard Controller provides a fixed message mapping, which is easy to implement, but not flexible enough to meet the needs of the system development and the user. Virtools provides a behavioral interaction module, Keyboard Mapper, to support mapping key operations into specified messages, which can be mapped to system messages, customize messages and mappings. The macro display of virtual reality and the specific performance of the operator are switched with the keyboard "C" key. The VSL of the scene transformation is shown in Figure 2.

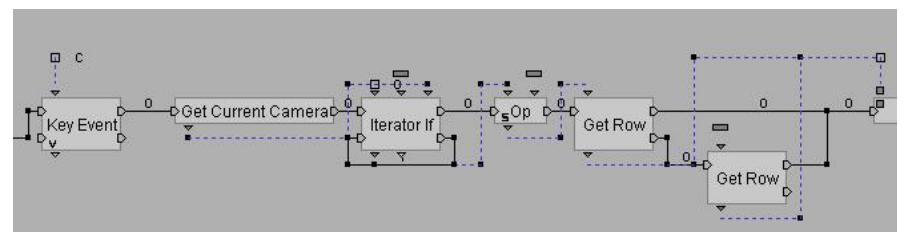

FIGURE II. SCENE CONVERSION DIAGRAM

In the specific sight mark display, we take the input "break armor" parameter as an example to introduce.

\section{A. Messages That Control Simulation}

Define The control between the various display interfaces of the control simulation is realized by the Virtools message processing mechanism. The mapping of system messages and custom messages to the specified actions is shown in Figure 3.
Using these interactive modules, the messages can be transferred flexibly between different objects in the control stream, and the virtual roles can be controlled flexibly according to user's habits and requirements.

Virtools provides a behavior interaction module Send Message, Send Message To Message, Wait Message, Broadcast Message support messages between different objects of different control streams, including system messages and custom messages. Accept. Send Message needs to specify the contents and recipients of the message. As shown in Figure 3, the system receives the bomb selection button through the keyboard and is pressed to start displaying the selected bomb species. The system sends messages to other modules after the last button is pressed.

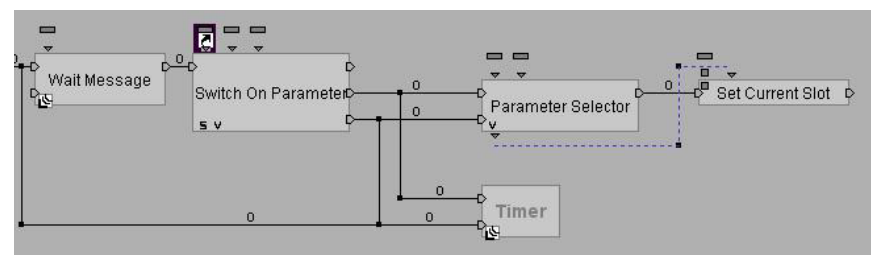

FIGURE III. PROJECTILE SELECTION MESSAGE RECEIVING

\section{B. Soft Switch Limitation Simulation}

Send message to message sends messages to the specified object group, while wait message and check for message complex check specified message get message data can read the data attached to the message. Dynamic connection with objects enables the whole system to run organically and orderly.

When the "Bomb seed" button is selected, when the mouse passes, the button state is changed and the message is released to the help interface, and the button prompt column is notified. When the bomb is selected as a "multipurpose bomb", the button operation VSL also sends the message to the console. Figure 4 is a VSL judgment function, which receives the message sent by the system, To make relevant judgments, select the next step to display the logo information in the sight window.

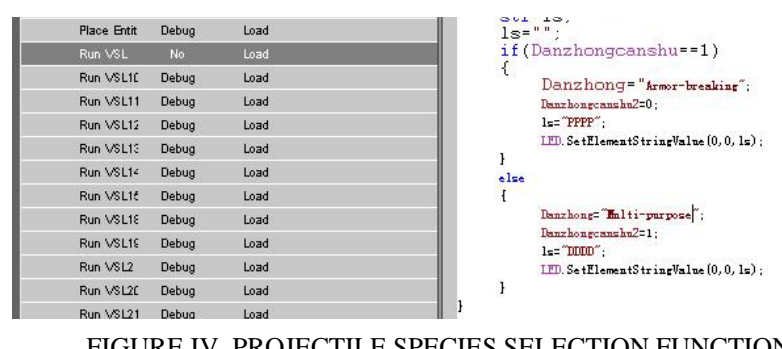

FIGURE IV. PROJECTILE SPECIES SELECTION FUNCTION 
C. Message Execution of Control Simulation

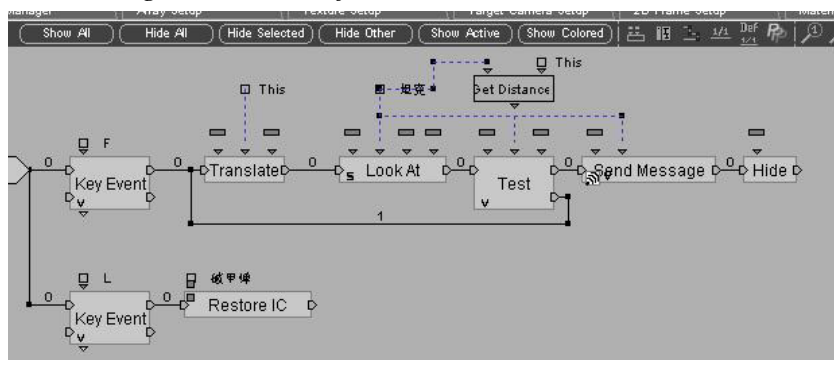

FIGURE V. PREPARATION OF MULTIPURPOSE PROJECTILESE

After the system console receives the message, on the one hand, by changing the system logo display parameters, change the symbol display content in Figure 1, on the other hand, notify the system loader to do the "multipurpose projectile" loading equipment.

The text instructions needed in the system are displayed through $2 \mathrm{D}$ text, which can reduce the system resources, make the system run more smoothly, create a two-dimensional frame in the system, and then carry on the text design to it. The BB that needs to be used is Creat System Font, Set Font Properfiles, and 2D Text.

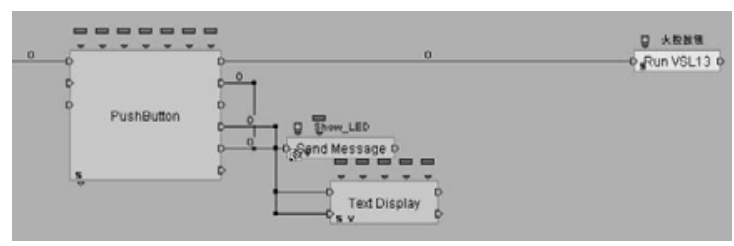

FIGURE VI. TEXT DESCRIPTION OF THE SYSTEM

\section{CONCLUSION}

The firepower simulation control training system is based on the mature computer multimedia technology in the design, absorbs the advanced simulation design idea, uses the standard computer peripherals as much as possible in the hardware design, in order to reduce the development difficulty and production cost. Besides, besides the training subject of the conventional air defense fire control system, it can also be based on the need. It is necessary to carry out a collaborative part of the training subject.

In this paper, from the bottom message transfer of Virtools, this paper deeply studies how to use the powerful function of VIRTOOLS and VC to realize the control function of the fire control system, which plays a core role in the complete completion of the simulation system. In the later period of the project, the two development of the higher level can be realized through the bottom communication, and the later project is expanded. The strength has been accumulated. Compared with traditional training, control simulation training has considerable advantages in improving training flexibility, breaking through training conditions and reducing equipment maintenance costs. Virtools software is very powerful and convenient to operate. It is very suitable for controlling the development of simulation system. In particular, it can improve the training quality, improve the training environment, improve the training environment and further improve the different content in training, aiming at the situation of the relatively expensive and complicated control system. A more realistic and practical simulation control training system has been developed.

\section{REFERENCES}

[1] T. M. Cheng, ”A fast parametric deformation mechanism for virtual reality applications”[J], Computer \& Industrial Engineering,2008,10(10): $18-26$.

[2] Jeffrey L Wampler, et. Integrating Maintainability and data development[C]. In: Proceedings of the Annual Reliability and Maintainability Symposium, Tamp Bay, 2003. 255-262.

[3] Pan Zhigeng, Cheok Adrian David. Virtual Reality and Mixed Reality for Virtual Learning Environments[J]. Computer \& Graphics, 2006, 30(1): 20-28.

[4] Daniel Cohen-Or. A survey of visibility for walk through applications[J]. IEEE Transactions on Visualization and Computer Graphics. 2003, 9(3): 412-431.

[5] Li J R, Khoo P. Tor B. Desktop Virtual Reality for Maintenance training An Object Oriented Prototype System(V-REALISM)[J]. Computerin Industry, 2003, 52(2): 109-125.

[6] Christopher M C, David C. Virtools Dev User Guide: Version 4.0[z]. Virwols SA, 2006.

[7] Virtools 5.0 Online Heferenee.

[8] Richens P,Nitsche M. Mindstage: Towards a ftmetional virtual architecture[C]. Computer Aided Architectural Design Futures proceedings, 2005: 331-340.

[9] Wutthikornthanawat w'Jinuntuya P,Rongviriyapanieh S, et a1. Multiuser tangible interface for public participation development of low-cost housing project design and planning[C]. Proeeedings of the 12th International Conference on Computer-Aided Architectural Design Research in Asia-Digitization and Globalizatin, 2007: 37-43.

[10] Wang Xi, An Yang. Development ofgame software based on Virtools[J]. Control and Management, 2007, 5(5): 160-162.

[11] Li Xunxiang, Chen Dingfang, Wang Le, et a1. A development framework for V'trtools-based DVR driving systemiC]. Computer Supported Cooperative Work in Design,2007: 188-196.

[12] Pan VLin Z,Hu Z, et a1. Research on distributed multi. screendis. play technique based on Virtools[C]. 7th Intemational Conferenee on Computer-Aided Industrial Design and Conceptual Design,2006: 1-6.

[13] D. U. Silverthom. Teaching and learning in the interactive classroom [J]. Advances in Physiology Education, 2006, 12(30): 135-140. 